\title{
The Validation of a Paddle Power Meter for Slalom Kayaking
}

\author{
(우)(1) (1) \\ Authors \\ Paul William Macdermid, Philip W. Fink \\ Affiliation \\ Massey University, School of Sport \& Exercise, Palmerston North, \\ New Zealand \\ Key words \\ slalom kayak, canoe, power meter, work \\ received 25.09.2016 \\ revised 07.11.2016 \\ accepted 18.12.2016 \\ Bibliography \\ DOI http://dx.doi.org/10.1055/s-0043-100380 \\ Sports Medicine International Open 2017; 2: E50-E57 \\ (c) Georg Thieme Verlag KG Stuttgart · New York \\ ISSN 2367-1890

\section{Correspondence} \\ Dr. Paul William Macdermid, MSc Exercise Physiology \\ Massey University \\ School of Sport \& Exercise \\ Private Bag 11222
}

\author{
4474, Palmerston North \\ New Zealand \\ Tel.: +64/6/951 6824, Fax: +64/6/9516824 \\ P.W.Macdermid@massey.ac.nz
}

\begin{abstract}
The ability to quantify power within the sport of slalom kayaking would provide coaches and athletes objective data to monitor performance. This study determined the validity of a kayak paddle power meter and subsequent relationship between work rate and stroke kinetics. One participant completed multiple trials of a flat-water, straight-line sprint and a sequence of slalom gates at numerous intensities to attain the velocity:power relationship. Laboratory results identified the kayak paddles' strain gauges were valid and reliable, and field tests presented a cubic relationship for power as a function of velocity in the straight-line sprint $\left(R^{2}=0.982\right)$ and the slalom-specific test $\left(R^{2}=0.993\right)$. Further analysis of stroke kinetics identified velocity of hand movement at individual peak force capability to be critical in the highest work rates achieved for both slalom and flat-water sprinting. It was concluded that the kayak paddle power meter tested is a valid means of recording work rate and stroke kinetics during kayaking in the field.
\end{abstract}

\section{Introduction}

Quantification of power output and work performed has proven crucial to the understanding of sports such as cycling $[2,3]$. The relationship between power and performance has been modelled to gain useful insights about elite cycling performance across the disciplines [7-9]. More recently [10], measurements of force during tethered linear sprints in slalom kayaks have been related to whitewater slalom performance. However, the ability to provide similar measures using this method during non-linear movements specific to slalom kayaking are not possible. Likewise, such difficulties are apparent in other sports due to technical and sport-specific limitations [6]. More recently, the advancement of strain gauge and accelerometer microtechnology with wireless functionality has led to the commercial development of power meters for water locomotion sports such as rowing (WebaSport, AUT) and kayaking (One Giant Leap, NZ). Although such pieces of equipment are very useful tools to monitor athletes in their natural environment, it is important they be validated.

The validation of bicycle power meters has been performed within a laboratory environment using dynamic calibration systems [3] and assessed within the ranges used by cyclists. Similar methods have more recently been performed with kayak paddles in a laboratory setting with regards to strain gauge validation [14] but excluding the kayaker-power meter interaction. Unlike in cycling, the exclusion of the human element is more difficult for kayaking, especially when the device is designed for use in the field and not a laboratory. However, the power output associated with flat-water locomotion has been found to be proportional to the boat velocity cubed $[1,12,18]$. This means that a reasonable validation protocol in the field for a device designed to log total power output whilst kayaking would involve an experienced kayaker, paddling at a variety of speeds in the same kayak, under the same environmental conditions. The resultant power output values plotted as a function of velocity would enable validity to be presented.

The kayak power meter (One Giant Leap, NZ) was primarily designed for flat-water kayaking but has recently been adapted to meet the specifications of slalom kayaking. This sport combines high degrees of physical, physiological and technical/strategic components $[5,11,16,17]$. Competitors race individually over a course of gates, negotiated in a set order and direction, whilst travelling down a whitewater river for a duration of roughly 90 s (International Canoe Federation Rules 2015). Training for this sport combines a multiplicity of methods focussing on a wide range of physical, physiological and psychological components [11]. With this in mind, the validation of a power meter specific for slalom kayaking could enhance the training environment afforded elite athletes and their support teams. 


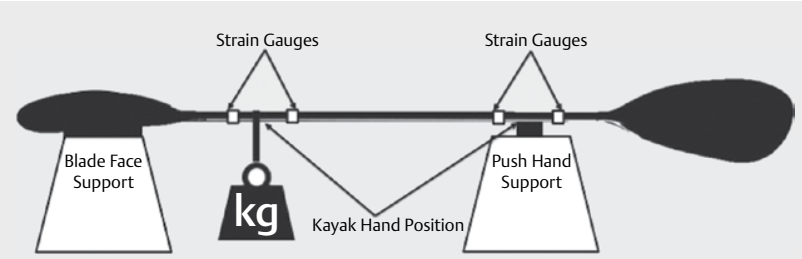

- Fig. 1 Pictorial image of the kayak paddle power meter set up for slalom kayaking.

Therefore, the primary purpose of this study was to determine the validity of a commercially available kayak paddle power meter in the laboratory through assessing strain gauge validity and in the field with a straight-line sprint and flat-water slalom course.

Based on models of rowing $[1,18]$ and kayaking [12], it was hypothesised that the power output required to propel the kayak would be proportional to the velocity cubed and thus prove validity of the equipment.

A secondary aim was to assess the relationship between power output and stroke kinetic metrics during flat-water, straight-line and slalom paddling.

\section{Method}

One experienced (30 yrs. national/international experience) male kayaker (age 43 yrs.; height $172 \mathrm{~cm}$; body weight including clothing, spraydeck and shoes $64.0 \mathrm{~kg}$ ) with extensive experience paddling with the power meter paddle (One Giant Leap, NZ), provided consent in accordance with the University Human Ethics Committee and the IJSM's ethical standards document [4]. The same kayak (Copa 2 Premium SL construction, medium size (length $350.5 \mathrm{~cm}$, width $60.8 \mathrm{~cm}$ ) Vajda, Bratislava, Slovak Republic) was used throughout. Because the main purpose of this study was to assess the validity of the kayak paddle rather than any test protocol, it was deemed more valuable to use one participant and thus control any inter-participant differences due to equipment and resultant drag effects on technique.

The power meter (One Giant Leap, NZ.), initially designed for flat-water sprint kayaking has subsequently been developed via firmware updates (version 3.17) for slalom kayaking. A split-shaft design enables $5 \mathrm{~cm}$ of paddle length adjustment and personal blade alignment angles to be set. The shaft has 12 load sensors located and offset around the circumference of the shaft at 4 different positions, on either side of the hand grip placement ( $\triangleright$ Fig. 1). This enables direct measurement of hand force over $360^{\circ}$ independent of blade pressure distribution and requires no assumption of the centre of pressure of the blade. Each shaft is fitted with an on-board inertial measuring unit that records hand acceleration and rotational velocity. Data from which is integrated into a commercially sensitive algorithm which enables the calculation of power within a stationary frame and from kinetic energy transferred to the paddle. Data is logged via a high speed mode $(60 \mathrm{~Hz})$.

Prior to commencing the field testing, strain gauge validity and reliability for both left and right sections of the shaft were individually determined under static conditions within a laboratory setting.
This involved hanging 3 known weights $(5.25,10.45$ and $15.89 \mathrm{~kg})$ from the reference point of the kayak hand position, with the drive blade supported on a solid surface while the opposing (pulling) hand position was also supported on a solid surface ( $\vee$ Fig. 1 ). These points were used because they are measurements required by the firmware algorithm used to determine power (http://support. onegiantleap.co.nz/getting-started.html). Each applied force was sampled 150 times in order to determine validity and reliability.

\section{Field test trial}

The participant attended a $2 \mathrm{~h}$ testing session which involved completing 2 tests on a lagoon, (Test 1: straight-line sprint; Test 2: slalom gates. See below for further explanation). Both tests were performed on a number of occasions (Test 1:30 times; Test 2: 20 times) at random intensities ranging from maximal effort to almost no effort and therefore eliciting a wide spectrum of velocities. Prior to commencing subsequent trials, the participant had recovered sufficiently not to affect technique. The tests were chosen in order to assess validity under normal forwards paddling stroke technique, typical of straight-line sprinting (Test 1, > Fig. 2a), and under slalom-specific conditions where strokes vary in order to negotiate a sequence of slalom gates (Test 2 , > Fig. $\mathbf{2} \mathbf{b}, \mathbf{c}$ ). The length of the session was $2 \mathrm{~h}$ to incorporate recovery time between each trial and time to download logged data because the power meter can store only 10 high-speed files at any one time. Environmental conditions included: water depth $2.2 \mathrm{~m}$; water temperature $9^{\circ} \mathrm{C}$; air temperature (shade) $13.9^{\circ} \mathrm{C}$; air temperature (sun) $38.9^{\circ} \mathrm{C}$; and wind speed $0.0 \mathrm{~m} \cdot \mathrm{s}^{-1}$.

Test 1 (Straight-line sprint, > Fig. 2a). From a seated stationary start, the participant began sprinting from a start buoy (Start, - Fig. 2a) and finished when crossing the threshold between 2 slalom poles (Finish, - Fig. 2a), a distance of $17 \mathrm{~m}$. The poles were $2 \mathrm{~m}$ in length, $1.2 \mathrm{~m}$ apart, and suspended $20 \mathrm{~cm}$ above the water (International Canoe Federation Slalom Rule 27).

Test 2 (Slalom gates, > Fig. $\mathbf{2 b}$,c). From a seated stationary start, the participant began sprinting from a start buoy (Start, - Fig. 2b,c), negotiated the set sequence of slalom gates (the poles were $2 \mathrm{~m}$ in length, $1.2 \mathrm{~m}$ apart, and suspended $20 \mathrm{~cm}$ above the water (International Canoe Federation Slalom Rule 27) and finished when crossing the threshold between 2 slalom poles (Finish, - Fig. $\mathbf{2 b , c )}$. The sequence of gates included 2 upstream gates and one downstream gate and equalled a total distance of $40 \mathrm{~m}$ travelled from start to finish.

\section{Outcome measures}

Performance time was assessed via high-speed $(300 \mathrm{~Hz})$ video footage (Casio Exilim Pro EX-F1, Japan) where the start position was determined as the body aligned with the start buoy and the right paddle blade first entered the water for the initial stroke. High-speed video analysis timing finished when the body crossed the line between the 2 slalom poles (Finish, > Fig. 2a-c). All performance time data for each trial was converted to average velocity per trial.

The kayak power meter logs force and power data for both left and right shafts separately at $60 \mathrm{~Hz}$. This can be transferred to a standard personal computer as a.csv file and processed using MATLAB R2014a. On a stroke-by-stroke basis, data were analysed for: A. Stroke length (s), defined as the time taken from data onset 


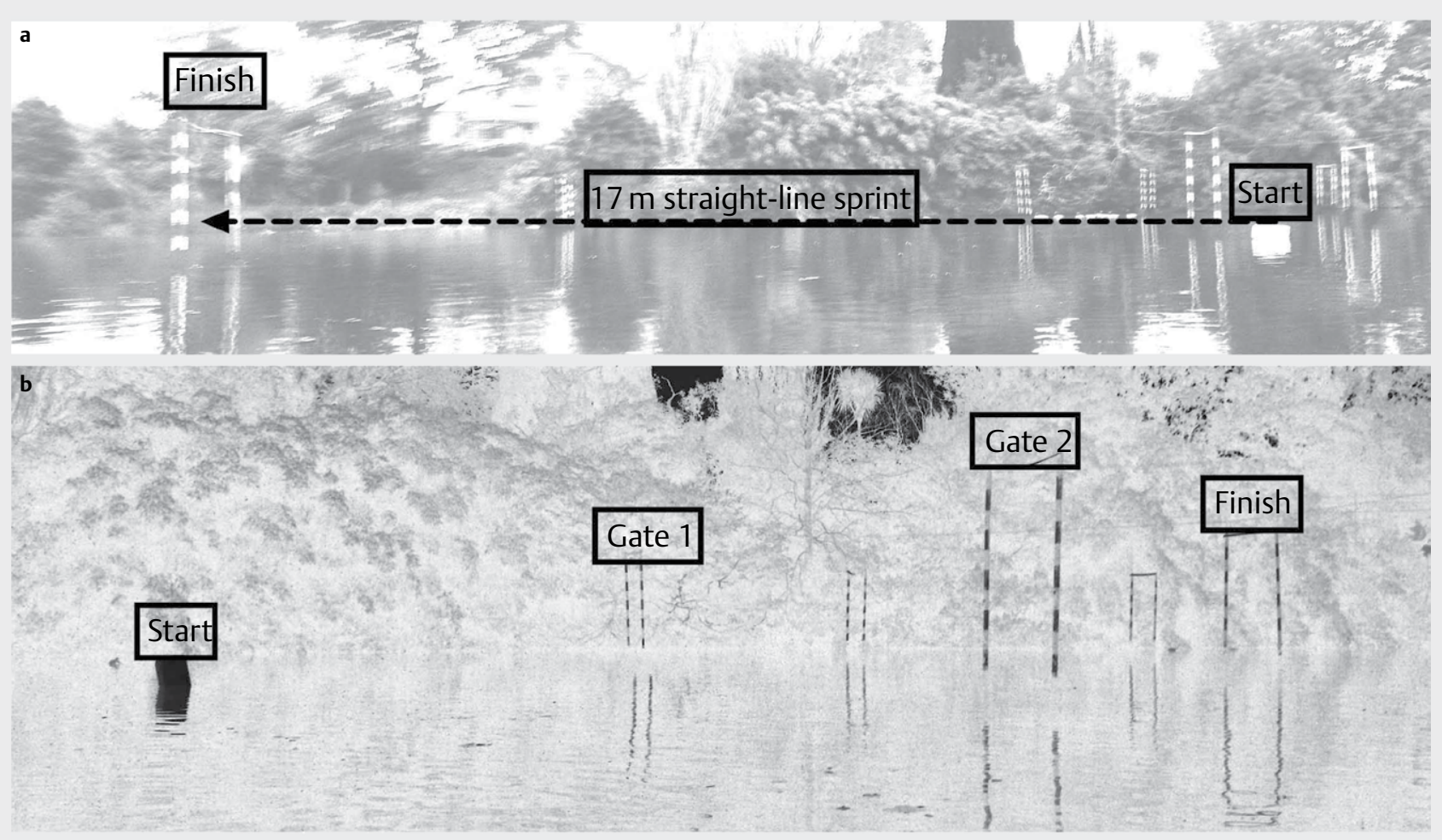

c

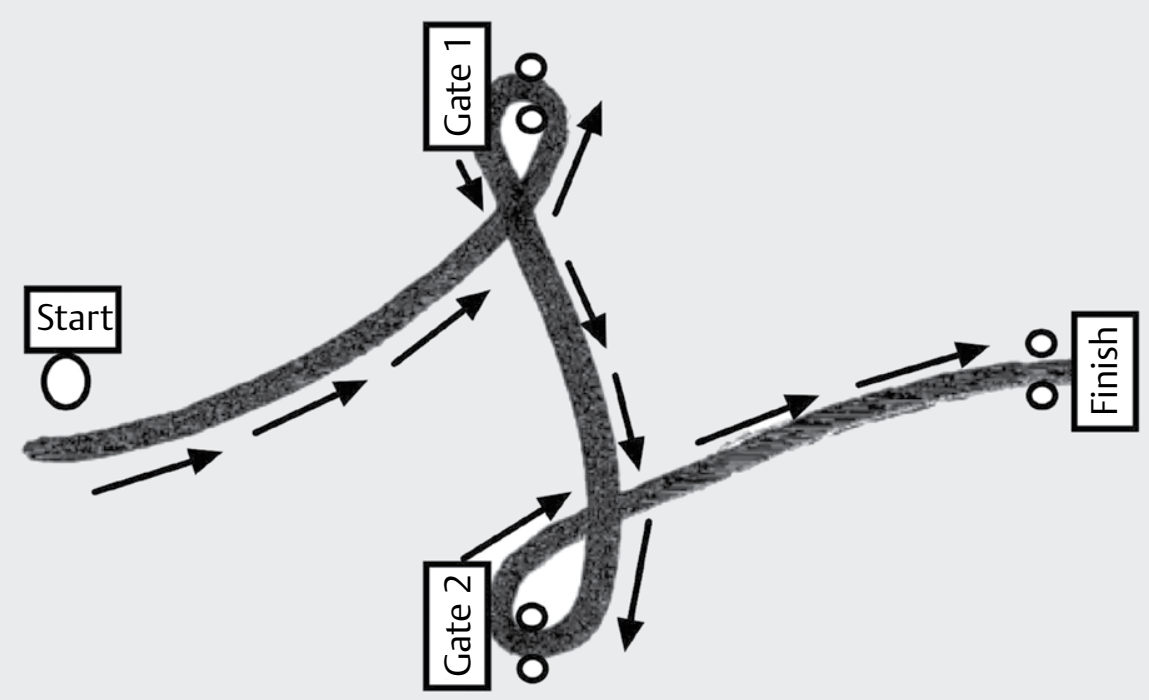

- Fig. 2 a Pictorial representation of the experimental protocol for the straight-line sprint (Test 1): Start signifies where the participant commenced the trial and the finish is where the participant crossed the threshold of the 2 slalom poles. $\mathbf{b}-\mathbf{c}$ Pictorial representation $\mathbf{b}$ of the experimental protocol of the slalom gate sequence (Test 2) and direction of travel c Start indicates the point at which timing and data logging commenced; gate number signifies the order of crossing indicated by the bold line; and the finish is where timing and accelerometry data logging ceased.

(when the drive side blade triggers a force threshold $>2 \mathrm{~N}$ and is $>10$ number points from the end of the previous stroke) to data offset (when the drive side blade triggers a force threshold <2 N); B. Impulse $\left(\mathrm{N} \cdot \mathrm{m}^{-1}\right)$, the area under the force curve per stroke; $\mathrm{C}$. Peak force $(N)$, the maximum force reached during each stroke; $D$. Time (s) to peak force, the time from stroke onset to the time of maximum force; E. Stroke rate (SPM), the number of strokes taken per minute; F. Drive (s), the time taken during which the paddle is producing propulsive force; $G$. Recovery (s), the time taken during which the paddle is not producing propulsive force; and H. Stroke ratio, the ratio of the recovery time to drive time. Subsequently, the data for the left and right shafts were combined and analysed for total power output at a sample rate of $60 \mathrm{~Hz}$ and averaged over 1 s epochs.

\section{Statistical analyses}

Descriptive (mean \pm SD, and range) data were calculated for all variables measured during each test. The relationship between 

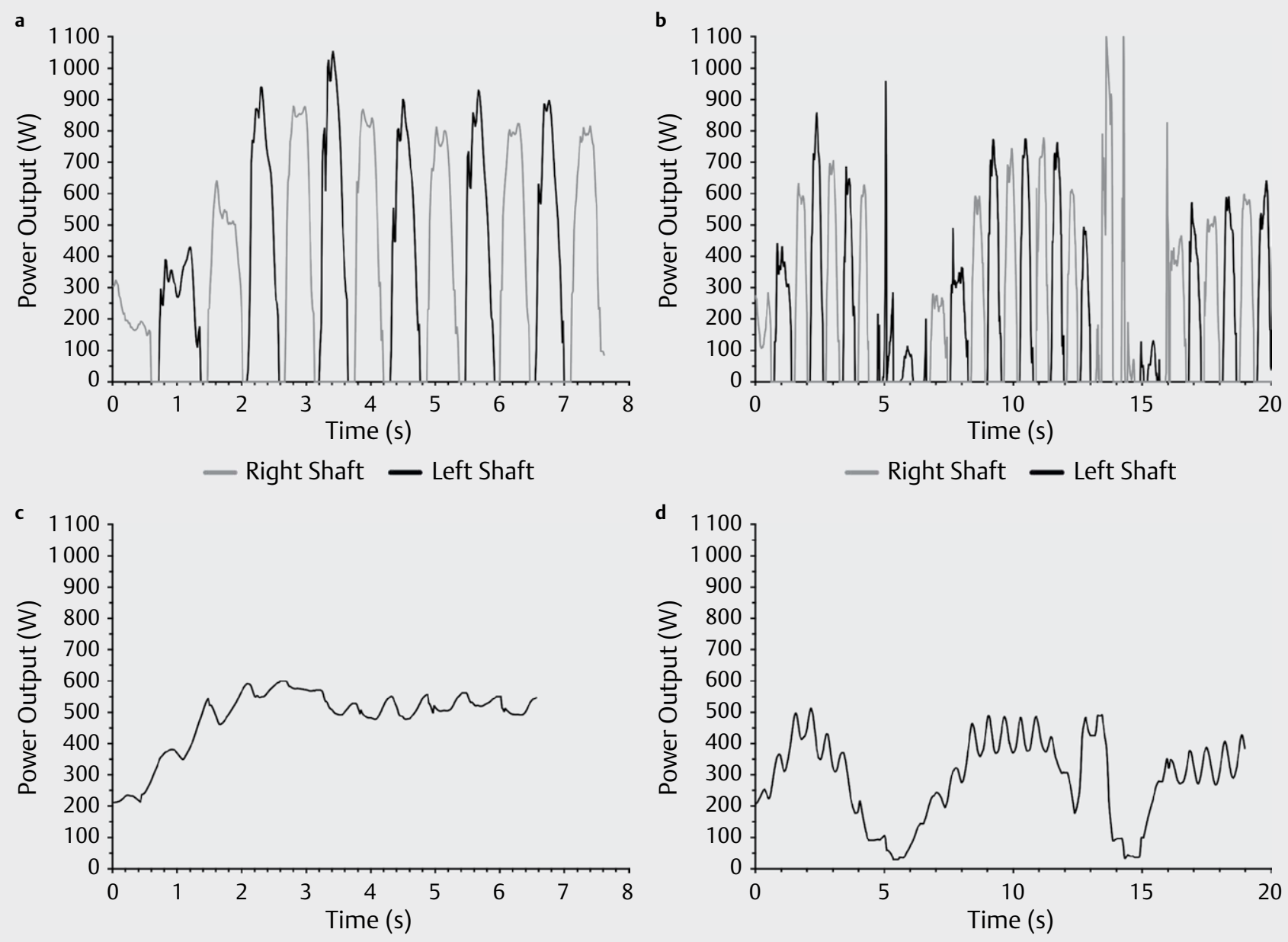

- Fig. 3 An example of an individual trial for Test 1 and Test 2. a Right and left paddle shaft power output for the straight-line sprint. b Total power output for the straight-line sprint. $\mathbf{c}$ Right and left paddle shaft power output for the slalom gate sequence. $\mathbf{d}$ Total power output for the slalom gate sequence.

power and velocity was tested through a non-linear fit regression on power, with the cube of velocity as the independent variable. To assess the goodness of fit, $r^{2}$ values were calculated between power and velocity cubed. All statistical analyses were performed using Graphpad Prism (V6.0f).

\section{Results}

Prior to commencement of the main research trial, validity and reliability of the strain gauges built into the left and right paddle shaft sections were assessed. Mean \pm SD for the 51.5, 102.5 and $155.9 \mathrm{~N}$ forces applied $(50.98 \pm 0.39$ and $50.74 \pm 0.75 ; 101.9 \pm 0.12$ and $101.6 \pm 0.44$; and $155.7 \pm 0.41$ and $154.7 \pm 0.52 \mathrm{~N}$ for the left and right shafts, respectively) highlights validity between $0.12-1.4 \%$. Reliability of each (left and right) shaft section expressed as a co-efficient of variation for the 51.5, 102.5 and $155.9 \mathrm{~N}$ forces applied equated to 0.76 and $1.48 ; 0.12$ and 0.44 ; and 0.27 and $0.34 \%$ per left and right shafts, respectively).

\section{Test 1 (straight-line sprint)}

The participant completed 30 trials of Test 1 (17 m straight-line sprint) at random intensities resulting in performance times ranging from $6.827-11.790 \mathrm{~s}$ with mean velocities of $1.442-2.490 \mathrm{~m} \cdot \mathrm{s}^{-1}$. This provided mean power outputs within the range of $47.2-$ 491.5 W. - Fig. 3a,b shows an individual trial of Test 1 highlighting power output for both the left and right paddle shafts ( $\vee$ Fig. $3 a$ ) and the accumulative $1 \mathrm{~s}$ average of both left and right shafts ( $\triangleright$ Fig. 3b). The relationship between velocity and power output over the straight-line sprint is shown in $>$ Fig. $\mathbf{4 a}$, along with the best fit line from the non-linear regression of velocity cubed against mean power $\left(R^{2}=0.982\right)$.

\section{Test 2 (slalom)}

Data analysis for Test 2 (a slalom-specific test), performed at random velocities $\left(1.409-2.164 \mathrm{~m} \cdot \mathrm{s}^{-1}\right)$ in an unspecified order, produced performance times between $18.48-28.39 \mathrm{~s}$. The resultant mean power outputs of these trials varied between $42.4-308.5 \mathrm{~W}$.

- Fig. $\mathbf{3 c , d}$ shows an individual trial of Test 2 highlighting power output for both the left and right paddle shafts ( $\vee$ Fig. 3c) and the accumulative $1 \mathrm{~s}$ average of both left and right shafts ( $\boldsymbol{\nabla}$ Fig. $\mathbf{3 d}$ ). A strong fit $\left(R^{2}=0.993\right)$ was also found between power and velocity cubed ( $\triangleright$ Fig. $\mathbf{4 b}$ ). 

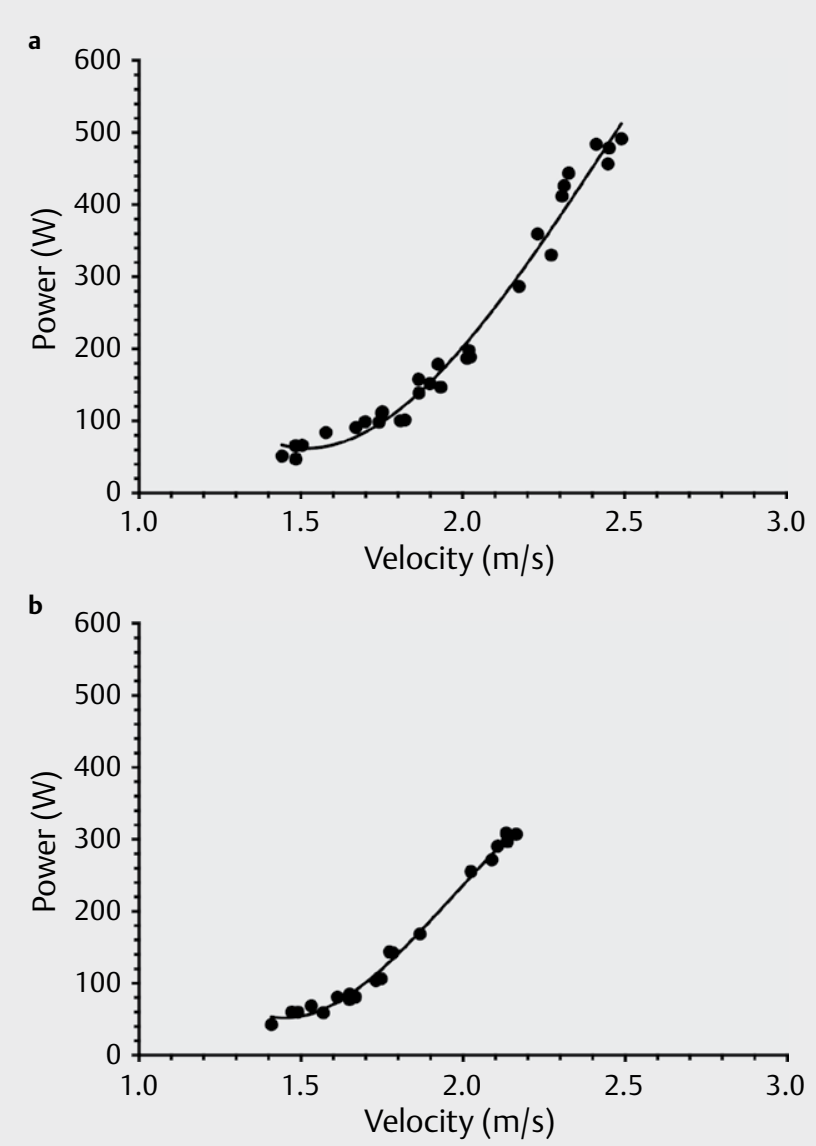

- Fig. 4 The relationship between velocity and power output for a The straight-line sprint and $\mathbf{b}$ The slalom-specific test.

\section{Other variables}

As there was a strong link between performance velocity and power output in both Test 1 and Test 2 ( $\triangleright$ Fig. 4a,b) further analysis presenting the relationship between power output and mean stroke by stroke metrics (drive time, recovery time, stroke rate, stroke ratio, impulse, peak force and time to peak force) are presented in

$\checkmark$ Fig. 5a-f and $\triangleright \mathbf{6 a - f}$, respectively.

\section{Discussion and Implications}

The primary aim of this study was to determine the validity of a commercially available kayak paddle power meter in a forwardssprint and a flat-water slalom course using similar stroke combinations to those faced during training and racing. The main findings show that: A. the kayak paddles strain gauges were valid and reliable; B. the kayak paddle power meter maintained a valid level of consistency with regards to power outputs logged over random velocities in a straight-line test; $C$. this relationship was mirrored when performing the slalom-specific test with varying stroke patterns; and D. kinetic metrics plateaued early in the power spectrum and the participant relied on velocity of hand movement for increases in power.

The kayak paddle shaft used within this study provided force exerted and hand movement velocities, enabling the calculation of independent (left and right stroke) power output at a rate of $60 \mathrm{~Hz}$ ( Fig. 3a,c). Subsequent analysis enabled smoothing to $1 \mathrm{~Hz}$ ( $\triangleright$ Fig. 3b,d) and the averaging over each trial to make a comparison with velocity of the kayak ( $\triangleright$ Fig. 4a,b). Initially the kayak paddle was designed for flat-water, straight-line events such as sprint or marathon. As such, the locomotory velocity of a kayak depends on the propulsive force (power) generated by the paddler [13]. Yet for movement or acceleration to occur, this force has to be greater than the combined resistive forces of active plus passive drag [12]. Consequentially, as speed increases the drag components and total energy costs increase, typically presenting a cubic relationship between velocity and power [15]. With no changes in boat direction or frontal surface area, and only using forwards paddling strokes, Test 1 ( $\triangleright$ Fig. 2a) was used to ascertain validity of this relationship. - Fig. 4a supports such a relationship and in so doing it is reasonable to assume validity of the power meter shaft for normal straight-line paddling. This offers an advantage over the only other method currently used in the sport of canoe slalom where paddlers were fixed to a complicated tethered system not available to individual athletes or coaches, and could only be used for sprints in a straight line [10]. However, a slalom paddler negotiates a sequence of gates, rarely travels in a straight line and can use a number of different strokes in any range of combinations. As such, the Newtonian principles of action-reaction (drag) still apply. Yet differences in the rate of force development and its relationship with velocity during a slalom-specific sequence was refuted due to the stroke(s) used and boat handling/positioning to negotiate gates. - Fig. 4b shows the relationship between velocity (performance) and power output to be comparable under quite different conditions (Test 1 , - Fig. 4a), but still on flat water. Therefore, the 2 tests used within this study ( $>$ Fig. 2) would provide the users of such scientific equipment with constructive information about performance and training adaptation, applicable to performance over a range of kayak disciplines including flat-water sprint and marathon, slalom, wild water racing and polo).

Although this study set out only to test the validity of the paddle to measure power output, it was believed pertinent for future studies to assess the relationship between work rate and stroke metrics. Although the data is limited because it can be applied only to one participant, it once again highlights the similarities between straight-line ( $>$ Fig. 5) and slalom ( $>$ Fig. 6) flat-water stroke kinetics and work rate achieved. Both trials saw kinetic metrics plateau early in the power spectrum, meaning further performance enhancement for this kayaker came from increased hand velocity or stroke frequency. Interestingly, the addition of a technical element by way of introducing the slalom gates sequence (Test 2) showed greater reliance on force rather than hand velocity to increase boat velocity compared to straight-line sprinting. Extrapolation at the $200 \mathrm{~W}$ work rate in $>$ Fig. 5,6 identifies reductions of the stroke frequency by $\sim 4-5 \%$ ( $\triangleright$ Fig. 5a, $\triangleright \mathbf{6 a}$ ) but a similar increase of $\sim 5 \%$ in peak force ( $\triangleright$ Fig. 5e, $\triangleright \mathbf{6 e}$ ) and impulse ( $\mathbf{F i g . 5 c}, \triangleright \mathbf{6 c}$ ). The authors surmise that this finding would be exacerbated further with increased difficulty of the course or whitewater to be negotiated. Because validation of the power meter has been presented, further research needs to establish the relationship between stroke kinetics and performance across a range of abilities, and whether it is useful to glean small changes in performance as a result of physi- 

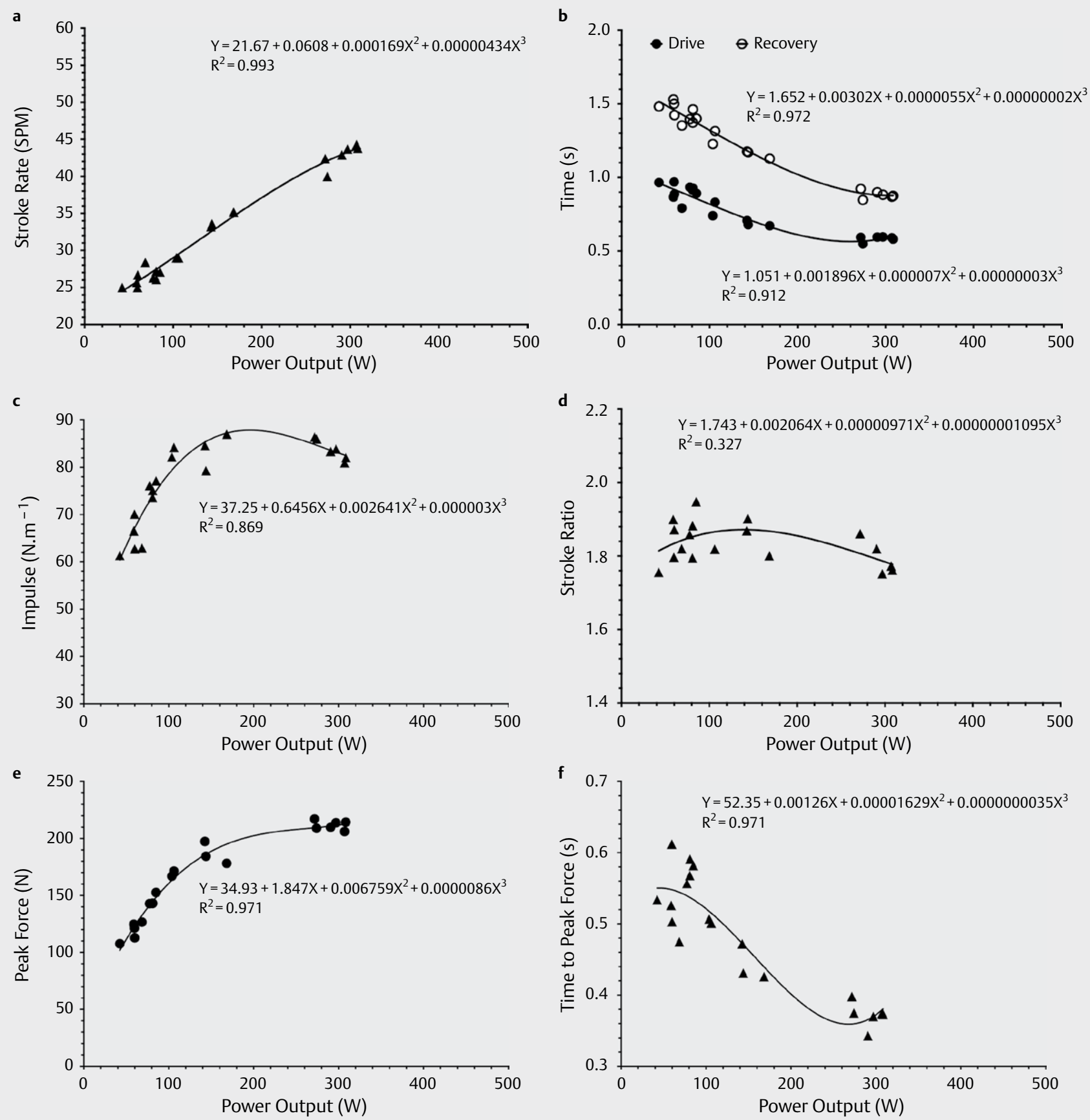

- Fig. 5 The relationship between power output (W) and a stroke rate (SPM); $\mathbf{b}$ drive and recovery time (s); $\mathbf{c}$ impulse ( $\left.\mathrm{N} \cdot \mathrm{m}^{-1}\right)$; $\mathbf{d}$ stroke ratio; $\mathbf{e}$ peak force $(\mathrm{N})$; $\mathbf{f}$ time to reach peak force $(\mathrm{s})$, taken as the mean of stroke by stroke data for the flat-water, straight-line sprint.

cal/technical enhancement that may occur amongst elite slalom kayakers.

\section{Conclusion}

This study set out to determine the validity of a commercially available kayak paddle power meter in a forwards-sprint and a flat-water slalom course using similar stroke combinations to those faced during training and racing. As such it has been shown that the pad- dles provide power outputs consistent with traditional models when plotted as a function of velocity in a straight line and whilst negotiating a specific course of slalom gates. Therefore, the device is a valid means of assessing work rate during kayaking and can be used in the field for research, performance monitoring and training prescription. Further research needs to be completed to assess the relationship between stroke kinetics and performance across a range of abilities. 

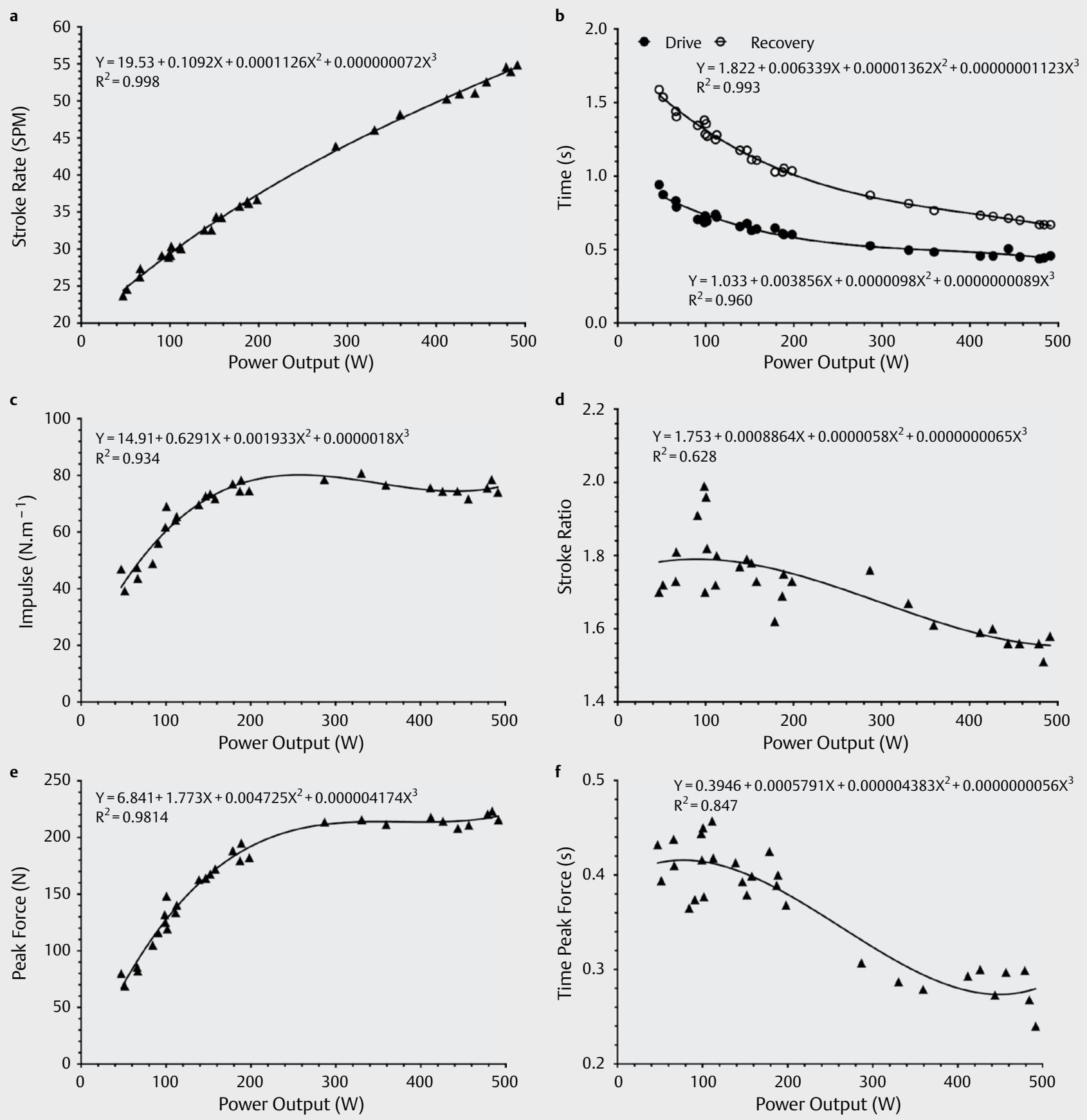

- Fig. 6 The relationship between power output (W) and a stroke rate (SPM); b drive and recovery time (s); $\mathbf{c}$ impulse $\left(\mathrm{N} \cdot \mathrm{m}^{-1}\right)$; $\mathbf{d}$ stroke ratio; $\mathbf{e}$ peak force $(\mathrm{N})$; $\mathbf{f}$ time to reach peak force $(\mathrm{s})$, taken as the mean of stroke by stroke data for the slalom-specific test.

\section{Conflict of Interest}

The authors declare that they have no conflict of interest.

\section{References}

[1] Cabrera D, Ruina A, Kleshnev V. A simple 1+ dimensional model of rowing mimics observed forces and motions. Hum Mov Sci 2006; 25: $192-220$

[2] Ebert TR, Martin DT, McDonald W, Victor J, Plummer J, Withers RT. Power output during women's World Cup road cycle racing. Eur J Appl Physiol 2005; 95: 529-536 
[3] Gardner AS, Stephens S, Martin DT, Lawton E, Lee H, Jenkins D. Accuracy of SRM and power tap power monitoring systems for bicycling. Med Sci Sports Exerc 2004; 36: 1252-1258

[4] Harriss D, Atkinson G. Ethical standards in sport and exercise science research: 2014 update $\bullet$. Int J Sports Med 2013; 34: 1025-1028

[5] Hunter A, Cochrane J, Sachlikidis A. Canoe slalom competition analysis. Sports Biomech 2008; 7: 24-37

[6] Kjendlie P-L, Thorsvald K. A tethered swimming power test is highly reliable. Port J Sport Sci 2006; 6: 231-233

[7] Macdermid PW, Stannard S. Mechanical work and physiological responses to simulated cross country mountain bike racing. J Sports Sci 2012; 30: 1491-1501

[8] Martin JC, Gardner AS, Barras M, Martin DT. Modeling sprint cycling using field-derived parameters and forward integration. Med Sci Sports Exerc 2006; 38: 592

[9] Martin JC, Milliken DL, Cobb JE, McFadden KL, Coggan AR. Validation of a mathematical model for road cycling power. Journal Appl Biomech 1998; 14: 276-291

[10] Messias L, Ferrari H, Sousa F, dos Reis I, Serra C, Gobatto C, Manchado-Gobatto F. All-out test in tethered canoe system can determine anaerobic parameters of elite kayakers. Int J Sports Med 2015; 94 : 803-808
[11] Messias LHD, dos Reis IGM, Ferrari HG, de Barros Manchado-Gobatto F. Physiological, psychological and biomechanical parameters applied in canoe slalom training: a review. Int J Perform Anal Sport 2014; 14: 24-41

[12] Pendergast D, Mollendorf J, Zamparo P, Termin A II. The influence of drag on human locomotion in water. Undersea Hyperb Med 2005; 32: 45

[13] Pendergast D, Zamparo P, di Prampero PE, Capelli C, Cerretelli P, Termin A, Craig A, Bushnell D, Paschke D, Mollendorf J. Energy balance of human locomotion in water. Eur J Appl Physiol 2003; 90: 377-386

[14] Sturm D, Yousaf K, Brodin L-Å, Halvorsen K. Wireless kayak on-water ergometry-Part 1: Paddle blade force. Sports Technology 2013; 6: $29-42$

[15] Wilson DG. Bicycling Science. Cambridge: MIT Press; 2004

[16] Zamparo P, Capelli C, Guerrini G. Energetics of kayaking at submaximal and maximal speeds. Eur J Appl Physiol Occup Physiol 1999; 80: 542-548

[17] Zamparo P, Tomadini S, Didone F, Grazzina F, Rejc E, Capelli C. Bioenergetics of a slalom kayak (K1) competition. Int J Sports Med 2006; 27: 546-552

[18] Zatsiorsky VM, Yakunin N. Mechanics and biomechanics of rowing: a review. Int J Sport Biomech 1991; 7: 229-281 